\title{
POLÍTICA COMERCIAL DE ESTADOS UNIDOS: UNA REVISIÓN DE LOS ALCANCES DE LA ADMINISTRACIÓN TRUMP
}

\author{
U. S. TRADE POLICY: A REVIEW OF THE SCOPE \\ OF THE TRUMP ADMINISTRATION
}

\author{
Gerald Solano Aguilar ${ }^{1}$ \\ ORCID: 0000-0002-7415-6003
}

\section{Jorge Rivera Hernández ${ }^{2}$ \\ ORCID: 0000-0003-4631-4091}

\section{Geovanny A. Blanco Fonseca ${ }^{3}$ \\ ORCID: 0000-0002-9167-2720}

\section{RESUMEN:}

Los parámetros de la política comercial de los Estados Unidos han experimentado una serie de cambios drásticos con la llegada del presidente Donald Trump a la Casa Blanca. Sus posturas proteccionistas, así como sus ataques en contra de los sistemas multilaterales que regulan el comercio internacional, han terminado por provocar un cambio sistemático en las relaciones comerciales de los EE. UU. El siguiente artículo recopila las posturas que antecedieron a la administración vigente, así como las prioridades y promesas realizadas por el presidente norteamericano debido a proteger los intereses nacionales de los EE. UU. De la misma forma, se realiza un análisis sobre los efectos y alcances de la política comercial llevada a cabo por Donald Trump en el inicio de su gestión.

Palabras clave: Donald Trump; efectos y alcances comerciales; Estados Unidos; política comercial.

\section{ABSTRACT:}

The parameters of United States trade policy have undergone a series of drastic changes upon the arrival of President Donald Trump to the White House. Trump's protectionist positions, as well as his attacks against the multilateral systems have caused a systematic

1 Universidad Nacional, Escuela de Relaciones Internacionales, Costa Rica. Académico-investigador, consultor y analista internacional. Responsable del Proyecto: Pulso de la Política Comercial de los Estados Unidos de América hacia América Latina. Colaborador en la Red Mexicana de Relaciones Internacionales y Desarrollo Global. Correo electrónico: gerald.solano.aguilar@una.cr

2 Universidad Nacional, Escuela de Relaciones Internacionales, Costa Rica. Profesor investigador y académico. Máster y especialista en Relaciones Internacionales, política internacional y negocios internacionales. Correo electrónico: jorge.rivera.hernandez@una.cr

3 Universidad Nacional, Escuela de Relaciones Internacionales, Costa Rica. Licenciado en Relaciones Internacionales con énfasis en Comercio Internacional y Egresado de la Licenciatura de Relaciones Internacionales con énfasis en Política Exterior y Diplomacia. Correo electrónico: giovannialexb23@ gmail.com 
change in the commercial relations of the North American nation. The following research paper compiles the positions that preceded the Trump Administration, as well as the priorities and promises made by the current US president in order to protect the national interest of the
United States. In the same way, the article reviews the effects and scope of the commercial policy carried out by Donald Trump during the beginning of his administration.

Keywords: Donald Trump; commercial effects and scope; United States; trade policy.

\section{Introducción}

Transcurría el 2015, cuando el afamado magnate Donald John Trump manifestaba sus intenciones de competir por la silla presidencial de los Estados Unidos de América (EE. UU.), en la campaña que iniciaría el año siguiente; no obstante, la llegada de este hombre tan mediático en un terreno tan volátil como la política, ya vislumbraba un panorama complejo, y el desarrollo de una campaña electoral atípica, y puede afirmarse que no decepcionó a nadie en este sentido. Es así, como después de un proceso marcado por hostilidades, y caracterizado por la polémica, terminaría siendo electo como el cuadragésimo quinto presidente de los EE. UU., el 8 de noviembre de 2016, al alcanzar la candidatura.

Este tema es de relevancia para las relaciones internacionales, en particular para las económicas, dado que al ser los EE. UU. la potencia hegemónica y principal fuente de economía global, las decisiones y acciones en materia de política internacionales, específicamente de política comercial, tienen impacto en el resto de los países. En este escenario es que, se busca responder: ¿cuáles han sido los alcances de la política comercial de los EE. UU. durante la era Trump?

Para responder a la incógnita, se realiza un análisis documental acerca de la política comercial de los EE. UU. durante las últimas administraciones (George W. Bush y Barak Obama), la evolución de los mecanismos que otorgan al presidente de los EE. UU. potestad para la toma de decisiones en materia de negociación internacional; así como lo alcanzado hasta el 2019. Para apoyar el estudio, se utilizan los postulados teóricos de Heckscher-Ohlin y la del neoliberalismo como forma de contextualizar y explicar las acciones del mandatario estadounidense en términos de política comercial. El presente trabajo pretende exponer los principales alcances de los EE. UU., el artículo se divide en tres partes: la primera aborda los antecedentes de la política comercial de los EE. UU., en una segunda parte se expone las criticas que esta ha recibido durante la administración Trump; la tercera y última, analiza los alcances logrados. 


\section{Antecedentes}

Las administraciones que antecedieron al presidente Donald Trump representan la antítesis de la posición del polémico mandatario. Hoy día, el proteccionismo marca el compás de la política comercial estadounidense, lo que fija un contraste con las posturas otrora liberales que, posicionaban a los EE. UU. como un socio comercial potencial para otras naciones. El autor Michael Wolff recopila en su libro Fire and Fury inside the Trump White House parte de lo que fue el discurso de inauguración del 20 de enero de 2017, en el cual, el nuevo presidente de los EE. UU. señala lo que será su posición, al anteponer su postura proteccionista, ya mostrada en campaña, y muestra su interés en proteger las fronteras estadounidenses de lo que él mismo denominó como: "estragos de otros países que fabrican sus productos, roban sus empresas y destruyen puestos de trabajo" (2018, p. 27). Para este autor, Trump ratificaba al pueblo norteamericano su vocación por patrocinar prosperidad y fuerza a través de una visión de protección que terminó por ofrecer un "triunfo para América", al manifestar su clara intención de recuperar el empleo y la riqueza para sus connacionales.

Por su parte, Robert Pollin (2005) expresa que, uno de los ejemplos de la postura de liberalización comercial recae en la apertura profesada por parte de George W. Bush, y cuya postura mostró una clara fidelidad a la doctrina de libre comercio, característica que siguió una línea similar a la posición asumida por el gobierno de Bill Clinton. Sin embargo, desde la perspectiva de Pollin, este paradigma le generó a Bush múltiples críticas, por cuanto se cuestionó su verdadera vocación liberal cuando impuso restricciones comerciales que respondían a intereses que tendían a favorecer su agenda política (2005, p. 127-128).

Es de esta forma que EE. UU. optó entonces, por promover la liberalización, y enfocar cada vez más sus esfuerzos en la búsqueda de iniciativas comerciales tanto de índole regional como bilateral. De allí que Crook (2003) también recalca el afán de fortalecer, por parte de la nación norteamericana, en instrumentos tales como el Área de Libre Comercio de las Américas (ALCA), el Tratado de Libre Comercio de América del Norte (NAFTA, por sus siglas en inglés), el Tratado de Libre Comercio entre República Dominicana, Centroamérica y Estados Unidos (CAFTA-DR, por sus siglas en inglés), además de acuerdos comerciales con Chile y Singapur.

Con esta liberalización, EE. UU. logró evadir la necesidad de consenso que convoca cualquier tipo de gestión a través de la Organización Mundial de Comercio (OMC); con lo cual el país norteamericano, era capaz de formular acuerdos con condiciones acatadas únicamente por las partes involucradas. Pero pronto se empezó a percibir un efecto adverso con esta postura liberal. 
En el nivel de América Latina, también se obtuvieron una serie de resultados importantes para la política comercial durante la era Bush. El Tratado de Libre Comercio entre Estados Unidos y Chile fue el primero concluido bajo la nueva Autoridad de Promoción Comercial (TPA) en 2002. Desde que el acuerdo entró en vigor en 2004, las exportaciones de bienes a Chile experimentaron un aumento contabilizado en USD 5.2 miles de millones y en tanto que las importaciones con este socio comercial aumentaron en USD 5.3 billones en 2008 (Direcon, 2015). De igual manera, concluir el CAFTA-DR, significó una acción clave de la política comercial de los EE. UU. dado que, posterior a su aprobación por parte del Congreso, dicho instrumento no solo terminó por regular el comercio de manera bidireccional, sino que también constituyó una herramienta para el fomento del comercio intrarregional, al consolidar la Iniciativa de la Cuenca del Caribe (ICC) en un marco jurídico, negociado en forma bilateral. Asimismo, se llevó a cabo un importante avance en torno a los acuerdos con Panamá, Perú y Colombia, en tanto que en el nivel de la región del Caribe se expandieron las posibilidades económicas a través de la ampliación de la ICC (USTR, 2008, p. 6).

La administración que antecede a Trump tuvo como protagonista a Barack Obama, quien durante la campaña electoral de 2008 en sus discursos no realiza ninguna mención importante al tema de política comercial o bien al término comercio, lo que generó una expectativa negativa en cuanto a las probabilidades de que el gobierno invirtiese gran cantidad de recursos en ello (SELA, 2009, p. 25). La agenda comercial del presidente Obama no solo se presentaba muy rígida, sino que incluso dio la impresión de que el comercio no era su prioridad. No obstante, la necesidad de apertura en una agenda como la estadounidense, determinó la pauta a seguir por el país, lo que responde a la trayectoria histórica de los EE. UU. por promulgar con la globalización, neoliberalismo e intercambio comercial. Al respecto, primeramente, y en cuanto a la TPA y los tratados pendientes, cabe decir que la propuesta de Barack Obama se centró en consultas exhaustivas al Congreso, para establecer las limitaciones que tendría.

En este sentido, se procedió a diseñar un plan de acción, que permitiese que el acuerdo comercial con Panamá avanzara, y al mismo tiempo trabajara, en los términos correspondientes, para los tratados con Colombia y Corea del Sur (SELA, 2009, p. 27). La ruta de esta política comercial en el segundo periodo presidencial de Obama, se evidenciada en cuatro ejes específicos, los cuales se detallan a continuación: 


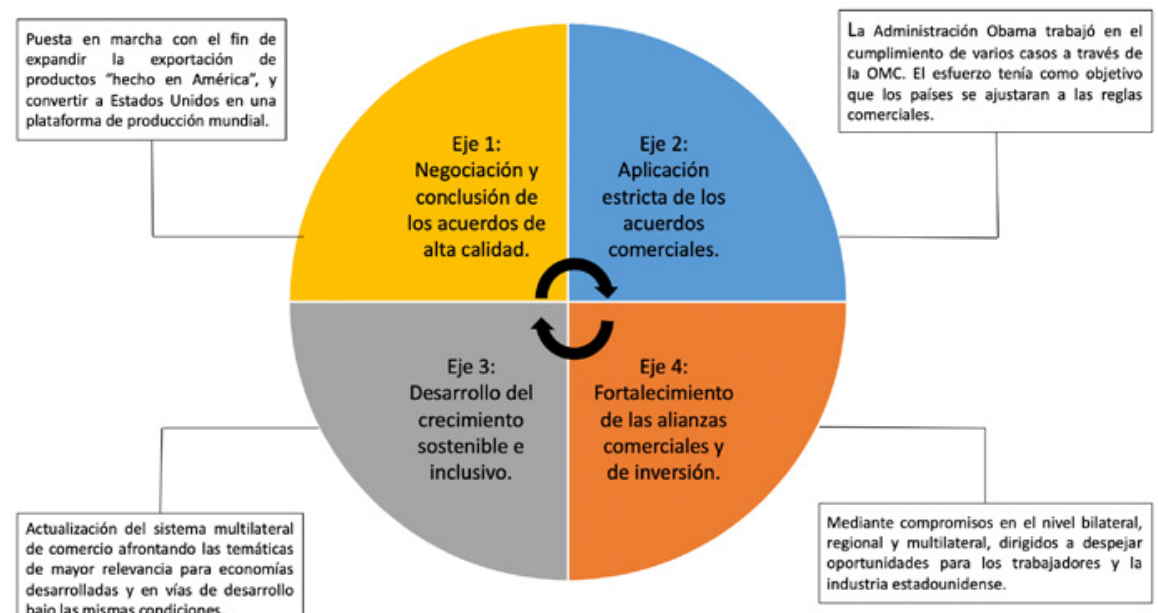

Figura 1. Ejes de la Política Comercial de la Administración Obama. Elaboración propia con base en Froman (2017).

Por otra parte, de acuerdo con Michael Froman (2017), todos estos ejes terminaron repercutiendo en el plano comercial, dado que Obama procedió con la formulación de una estrategia de carácter multifacético frente a la crisis económica, misma que estuvo constituida por un estímulo fiscal, así como por programas de rescate de la industria automotriz estadounidense, y reformas al sistema financiero; por lo que los EE. UU. reportaron una baja de USD $259 \mathrm{mi}-$ les de millones en sus exportaciones, "siendo esto la mayor pérdida en términos monetarios desde 1938" (p. 3).

Por lo anterior, en 2009 los EE. UU. en su capacidad de hegemón logró, a través del G-20 desalentar posturas con carácter proteccionista, que evitó recrudecer la situación, y contribuir a conservar empleos mientras se apoyaba el crecimiento; que conscientes o no, según Méndez "lo que hacía falta para el reconocimiento mundial de estas economías emergentes era una crisis que hiciera ver que el cambio institucional era necesario -y en 2008 esa crisis arribó-" (2016, p. 25). De acuerdo con el especialista en relaciones internacionales:

esta pugna o hegemonía material e ideacional se refleja en las instituciones internacionales que son, en buena medida herramientas para asegurar, estabilizar e incluso - como se indicó - absorber los cuestionamientos que enfrenta un orden mundial determinado y ensanchar la capacidad del hegemón para gestionar sus desafíos. (Méndez, 2016, 18) 
Estas medidas permitieron que EE. UU. lograra revertir los efectos de la crisis y posicionar su economía hacia un panorama de crecimiento y un estado más próspero al lograr que las empresas estadounidenses generaran 15.6 millones de nuevos empleos desde principios de 2010, en tanto que la generación de valor agregado manufacturero real aumentó $12.6 \%$ desde el punto más bajo de la recesión en el primer trimestre de 2009 , tendencia que se mantuvo hasta el segundo trimestre de 2016; además de crear 807000 nuevos puestos de trabajo en el sector manufacturero, desde su punto más bajo a principios de 2010. (Froman, 2017, p. 3)

Por lo anterior, puede decirse que la siguiente administración encontraría una economía más fuerte y robusta, acompañada además de un panorama comercial positivo, con respecto al que debió afrontar el expresidente Barack Obama en su primer año de gobierno. No obstante, la entrada en escena de Donald Trump como candidato a la Presidencia por el Partido Republicano, y su retórica marcada por el aborrecimiento a la gestión de su predecesor, forjaban sendas dudas respecto a asumir posturas liberales en el ámbito comercial; en especial, las aseveraciones de la campaña presidencial de 2016, cuando le dice a los votantes, que terminaría con la participación de EE. UU. en la Asociación Transpacífico, al aducir que su país no tiene la necesidad de suscribir ningún acuerdo internacional masivo.

De igual forma, se propuso la renegociación del NAFTA, con la amenaza incluso de retirar a EE. UU. en caso de ser necesario (USTR, 2018). En fin, Donald Trump ha impuesto un sello propio en lo que parece representar una nueva etapa de la política comercial estadounidense, centrándose en participar en un mercado global más justo en favor de los trabajadores estadounidenses, detalles que se abordarán en el siguiente apartado.

\section{Política comercial de la Administración Trump}

Como se ha señalado en líneas anteriores, el presidente Trump ha ejecutado acciones que conllevan implicaciones en la política comercial de los EE. UU.; por ello, es preciso indicar que se entiende la política comercial como:

una de las políticas económicas de mayor difusión entre las aplicadas por los gobiernos centrales de toda nación. Además de las tradicionales políticas fiscales y monetarias, los gestores de la política económica cuentan con la posibilidad de inducir comportamientos comerciales internacionales a través de la política comercial externa. La misma permite entrelazar el sistema productivo con los mercados foráneos a través de la apertura 
de la economía, los acuerdos comerciales y/o los procesos de integración económica regional. Existe un cierto consenso acerca de que una política comercial tendiente a la inserción de la economía nacional en los mercados internacionales constituye uno de los motores impulsores del crecimiento y desarrollo económico. (Bellina y Frontons, 2012)

Ahora bien, el presidente Donald Trump durante su campaña presidencial, planteó en sus discursos que los esfuerzos de su gobierno se mantendrían enfocados en la protección de los derechos comerciales de los EE. UU., así como de los trabajadores estadounidenses. Desde la rama republicana se ha manifestado que la política comercial llevada a cabo por el país antes de la llegada del actual presidente no era saludable, según se aducía, no porque no se crea en el libre comercio y en los mercados abiertos, sino porque no todos ven claros beneficios de los acuerdos comerciales; por lo que Trump terminó por adoptar un nuevo enfoque, y así cumplir con las promesas proteccionistas de campaña (O’Grady, 2018).

Por lo anterior, se procedió con formular una política comercial cuyo precepto principal se enfocaba en expandir el comercio más justo para todos los estadounidenses. Según la USTR de los EE. UU., esta postura comercial fue diseñada con el fin de aumentar el crecimiento económico, crear empleos, suscitar la reciprocidad con los socios, fortalecer la base de industria estadounidense, así como vigorizar la capacidad para defender y expandir las exportaciones de la industria agrícola y de servicios. Una de las diferencias con las políticas comerciales ejercidas por parte de las administraciones anteriores, yace en la creencia de que los objetivos propuestos pueden ser alcanzados a través de negociaciones bilaterales en lugar de multilaterales, al igual que al renegociar los acuerdos comerciales cuando las metas no se están cumpliendo (USTR, 2017, p. 13).

Es entonces que la administración Trump, propone los siguientes objetivos de política comercial, los cuales se enumeran a continuación:

- Asegurar que los trabajadores y las empresas de EE. UU. tengan una oportunidad justa de competir por negocios, tanto en el mercado doméstico, como en otros mercados claves de todo el mundo.

- Derribar las barreras comerciales injustas que bloquean las exportaciones estadounidenses en otros mercados, incluyendo las exportaciones de bienes agrícolas.

- Mantener una política equilibrada que defiende los intereses de todos los segmentos de la economía incluida la fabricación, la agricultura y los servicios, así como las pequeñas empresas y los empresarios. 
- Garantizar que los propietarios de propiedad intelectual en EE. UU. tengan una oportunidad plena y justa de usar y sacar provecho de esta.

- Hacer cumplir estrictamente las leyes comerciales de los Estados Unidos para evitar que el mercado doméstico se distorsione por dumping o importaciones subvencionadas que perjudican a las industrias y a los trabajadores nacionales.

- Hacer cumplir las disposiciones laborales en los acuerdos existentes, y la prohibición contra la importación y venta de bienes hechos con trabajo forzoso.

- Resistir los esfuerzos de otros países o miembros de organismos internacionales como la Organización Mundial del Comercio de avanzar en interpretaciones que debiliten los derechos y beneficios, o bien, aumentar las obligaciones en virtud de los diversos acuerdos comerciales en que Estados Unidos es parte.

- Actualizar los acuerdos comerciales actuales según sea necesario para reflejar los tiempos cambiantes y las condiciones del mercado.

- Asegurar que la política comercial de los Estados Unidos contribuya al fortalecimiento económico y a la fabricación base necesaria para mantener y desarrollar la seguridad nacional.

- Abogar enérgicamente por todos los trabajadores, granjeros, rancheros, proveedores de servicios y empresas de los Estados Unidos, grandes y pequeños: para garantizar el tratamiento más justo posible de los intereses estadounidenses en el mercado doméstico y en todo el mundo (USTR, 2017, p. 14).

Por lo anterior, se han identificado dos desafíos principales con respecto a las dinámicas comerciales que se perciben en el mercado global. El primero estriba en que las reglas de la OMC, así como de algunos acuerdos, tanto bilaterales como multilaterales, a menudo se formulan con un sentido implícito de que los países que efectúan dichos reglamentos siguen los principios del libre mercado. Adicionalmente, se abordan aspectos como la redacción, la implementación y la aplicación de las reglas, que deben encontrar formas de ajustarse a los principales temas comerciales de interés para los EE. UU. 
Por otra parte, el segundo desafío resalta que las reglas de la OMC y de los acuerdos comerciales, a menudo son formulados con la idea de que los países que las implementan gocen de sistemas legales y regulatorios funcionales y transparentes. No obstante, la transparencia en los sistemas es de suma importancia para el funcionamiento de las normas, dado que permite a las partes interesadas y a los gobiernos encarar los desafíos de índole diplomática y legales relativos a dichos reglamentos, que anticipen el momento en que no respondan a las obligaciones internacionales.

En este sentido, la administración Trump determina la necesidad de un enfoque más agresivo, echando mano de toda la influencia posible para alentar a otras naciones a otorgar a los productores estadounidenses la posibilidad de accesos equitativos y recíprocos en sus mercados, por ejemplo, la Unión Europea o el caso de China. Con este esfuerzo, se pretende garantizar la apertura de los mercados de bienes y servicios domésticos, reservándose la posibilidad de restringir el comercio y la competencia mundial, al tiempo que contribuye con el crecimiento de la economía al romper barreras comerciales (USTR, 2017, p. 17).

Otro frente de acción en materia comercial tenía como escenario los programas de trabajo de la Agenda de Doha para el Desarrollo. En lo referente al grupo de trabajo sobre comercio, deuda y finanzas, EE. UU. mantuvo un enfoque centrado en los aspectos de financiamiento comercial. En otro punto, con respecto al grupo de trabajo sobre comercio y transferencia de tecnología, la no calendarización de reuniones de este durante el primer año de la Administración dejó un panorama incierto tanto sobre la agenda de dicho grupo, así como por la postura asumida por los EE. UU.

En cuanto al programa de trabajo sobre comercio electrónico, EE. UU. manifestó su interés de trabajar junto con otros miembros con el fin de mantener un entorno comercial liberal para los productos y servicios que se comercializan electrónicamente, buscando garantizar que las normas sean apropiadas y justas con respecto a la economía digital. No obstante, esto dependerá del Consejo General, el cual continuará evaluando el progreso del programa de trabajo y considerará cualquier recomendación, incluso aquellas relacionadas con el estado de la moratoria de los derechos de aduana en las transmisiones electrónicas (USTR, 2017, pp. 33-35). 


\section{Promesas de Trump en su política comercial}

Desde la campaña electoral, Donald Trump se encargó de proponer una serie de medidas enfocadas a proteger los derechos comerciales de los EE. UU. Para efectos de este trabajo, se revisaron los discursos de campaña, ideales, y los prolegómenos de la política comercial, elementos que fueron analizados en el marco de la campaña del presidente y que se considera que lo colocaron en la Casa Blanca. De lo anterior, se han identificado al menos siete promesas que resultan puntos estratégicos de la agenda de la actual administración.

La primera, la intención del presidente norteamericano de retirar a los EE. UU. del Acuerdo Transpacífico de Cooperación Económica (TPP, por sus siglas en inglés). Trump procedió con el retiro de su país en su primer día en el cargo, dado que el acuerdo aún estaba pendiente de ratificar. Este hecho marca un hito, ya que es la primera vez en que EE. UU. se retira de un acuerdo comercial que previamente había defendido, a lo que, de acuerdo con la $\mathrm{BBC}$, el presidente Trump manifestó: “(El TPP) es un desastre potencial para nuestro país, . . . En cambio, negociaremos acuerdos comerciales bilaterales que generen empleos e industria en EE. UU. otra vez" (BBC, 2017, párr. 4).

Por otro lado, la intención de Trump residía en aumentar su influencia sobre ciertos países del TPP con los que carecía de algún acuerdo de libre comercio preexistente. La semana posterior a la salida del TPP, el presidente norteamericano manifestó su deseo de iniciar conversaciones bilaterales con Japón, al tiempo que de manera paradójica repitió sus quejas sobre las prácticas desleales llevadas a cabo por el país asiático debido a las importaciones de automóviles de los EE. UU. (France24, 2018). Los temas arancelarios entre EE. UU. y Japón fueron objeto de intensas negociaciones durante el proceso del TPP, algunos de los cuales se resolvieron en un anexo especial del acuerdo, aún y pese a no estar claro si EE. UU. esperaba que dicho anexo pudiera llevar a un acuerdo bilateral (DIIS, 2017, pp. 15-16).

Cabe resaltar que previo a la cumbre del presidente Trump con el primer ministro Japonés Shinzo Abe, el 10 de febrero de 2017, los funcionarios japoneses sugirieron que las negociaciones bilaterales eran poco probables, aunque en la cumbre, decidieron poner en marcha un diálogo intersectorial en torno al comercio y a la política, la cual fue dirigida por el vicepresidente de los EE. UU., Mike Pence, y el viceprimer ministro de Japón, Taro Aso (EFE, 2017). En los casos de Malasia y Vietnam, en lugar de presionar por acuerdos de libre comercio, EE. UU. únicamente se limitó a generar reclamos en el área comercial; no obstante, en una reunión realizada en septiembre de 2017, el Ministro de Comercio e Industria de Malasia, Mustapa Mohamed fue presionado para revisar 
la gestión con respecto a las exportaciones estadounidenses de productos agrícolas y otros bienes, así como el acceso de empresas estadounidenses a Malasia, mercados de seguros y servicios financieros, además de proteger la propiedad intelectual estadounidense, al cubrir de manera total los temas contenidos en el TPP (VOA, 2017).

En lo referente a Vietnam, se procedió con una reunión para presionar por eliminar la cuarentena en los destiladores de granos secos de los EE. UU., así como restaurar las normas del "Codex Maximum Residue Level" para medicamentos veterinarios utilizados por la industria ganadera estadounidense. Vietnam procedió con la realización de dichos cambios en septiembre de 2017 (DIIS, 2017, pp. 16-17).

La segunda promesa en materia comercial tiene que ver con el nombramiento de negociadores comerciales y de reconstruir la política comercial. Muy pocas cosas parecían seguras con la puesta en marcha de la política comercial de esta administración, entre esto, cabe mencionar la incertidumbre que provocaba la ausencia del Congreso en algunos de los detalles de su formulación, así como la carencia de liderazgo de los Republicanos y Demócratas de cualquier rol importante al respecto desde enero de 2017 (La Nación, 2017). Lo anterior, por cuanto el Partido Republicano demostró una postura contraria a la manifiesta por el presidente Trump, en particular sobre el descenso desde la campaña presidencial del comercio.

No obstante, cuando el nombramiento de Robert Lighthizer, como representante en materia de comercio para la renegociación de acuerdos comerciales, llegó al Senado en mayo de 2017, solamente tres senadores republicanos votaron en contra de él. En julio de 2017 los líderes demócratas en el Congreso procedieron con el lanzamiento de un nuevo programa político, denominado "a better deal", sobre comercio y empleos, el cual aborda las medidas enérgicas propuestas contra los países que manipulan las reglas comerciales, además de las penalizaciones a las empresas que subcontratan trabajos estadounidenses (DIIS, 2017, p. 19).

La tercera promesa de Donald Trump recae en identificar violaciones a los acuerdos comerciales con el fin de finalizar con los abusos. En términos más exactos, se trata de abordar las violaciones comerciales percibidas, abusos y lesiones llevadas a cabo por parte de socios comerciales, mediante la aplicación de una serie de herramientas bien establecidas que estarían disponibles para el ejecutivo.

Para ello, algunas de estas herramientas son los derechos antidumping, los derechos compensatorios, las salvaguardias, los aranceles, la suspensión de 
preferencias comerciales de los socios (en el caso de los programas de preferencias comerciales), el uso de consultas de un país a otro para resolver disputas bilaterales, enmendar acuerdos bilaterales, o bien, forjar otros más ventajosos; que aboguen por el inicio de un procedimiento de solución de diferencias en los acuerdos comerciales en los que están previstos, al mantener la excepción en los procedimientos de solución de diferencias de la OMC, sobre los cuales la agenda comercial de la administración Trump ya tenía reservas. Cabe decir que un aumento de la gestión de los EE. UU. en estas áreas podría haberse esperado; sin embargo, esta recae en la revisión de cada herramienta, con el fin de tutelar su debido cumplimiento (DIIS, 2017, p. 21).

De conformidad con la legislación comercial de los EE. UU., se puede inferir que las investigaciones y medidas antidumping, compensatorias y de salvaguarda pueden iniciarse por medio de peticiones de empresas estadounidenses, organismos comerciales o sindicatos al Departamento de Comercio o la Comisión de Comercio Internacional. El Departamento de Comercio lleva a cabo el elemento externo de las investigaciones relacionadas con las denuncias de dumping y de los exportadores que se benefician de los subsidios de gobiernos extranjeros. La Comisión de Comercio Internacional se encarga de investigar a lo interno de los EE. UU. para determinar si una industria estadounidense ha sufrido daños importantes por las mismas distorsiones presentes en el comercio. Por tanto, las definiciones de dumping, subvención y daño complementan la legislación comercial de los EE. UU., de conformidad con lo establecido en los artículos pertinentes de la OMC (DIIS, 2017, pp. 21-22).

En lo referente a la suspensión de las preferencias comerciales, EE. UU. mantiene tres programas en virtud de los cuales, los países en desarrollo reciben acceso libre de aranceles y contingentes al mercado estadounidense. Por lo general, a los países menos adelantados se les otorgan preferencias más generosas que a los países en desarrollo. El más grande de estos programas es el Sistema de Preferencias Generalizadas de Estados Unidos, el cual acoge alrededor de 120 países. Otros programas son la Ley de Crecimiento y Oportunidad para África (AGOA) y la Ley de Recuperación Económica de la Cuenca del Caribe (CBERA), los cuales cubren alrededor de cuarenta y once países, respectivamente.

El mayor beneficio comercial otorgado por la elegibilidad para estos programas se refiere a la vestimenta y los textiles de los países menos adelantados, que de otro modo se enfrentan a considerables barreras arancelarias y no arancelarias en los EE. UU., así como a las reglas de origen. Por lo general, los criterios de elegibilidad para estos programas, incluyen niveles de desarrollo económico, gobernanza, y los derechos de propiedad intelectual, además de los derechos humanos, los derechos de los trabajadores, la apertura a las exportaciones de 
los EE. UU. (aunque no necesariamente la provisión de acceso recíproco); y el empleo de sistemas que permitan monitorear las exportaciones de los países beneficiarios con las condiciones del programa, en especial en cuanto al origen (Steinberg, 2018).

Sobre el inicio de un procedimiento de solución de diferencias en los acuerdos comerciales, los países utilizan estos con el fin de remediar lo que consideran un trato injusto de sus exportaciones. Una de las promesas más importantes a considerar, es el tema de la renegociación del NAFTA, o bien, el retiro de este en caso de no llegar a un acuerdo con Canadá y México. En ese momento, Robert Lighthizer informó a la prensa que el deseo de los EE. UU. era renegociar el NAFTA sobre una base trilateral, aunque también se considerarían las negociaciones bilaterales si las negociaciones trilaterales fracasaban (New York Times, 2018).

Otra de las promesas de Trump se basaba en etiquetar a China como manipulador de divisas y tomar las contramedidas correspondientes. La intervención del Gobierno en los mercados de divisas para evitar que las divisas suban frente al dólar estadounidense ha sido vista por una amplia gama de economistas y formuladores de políticas estadounidenses como la manipulación de divisas (CNN, 2019). Se considera que responder a dicha acción requiere una postura firme, ya que una tasa de cambio subvaluada puede ser considerada tanto un impuesto a la importación, como un subsidio a la exportación, por lo que se convierte en una política más mercantilista.

La sexta promesa tiene que ver con el abordaje de los casos de comercio en contra de China. Con la manipulación de divisas en un segundo plano, EE. UU. procedió a actuar en tres frentes de política comercial. Al menos uno de ellos puede verse como una forma de presionar, que también se está intensificando en la OMC, el cual apunta hacia las investigaciones antidumping, y en materia de derechos compensatorios, así como aplicar derechos para determinar los márgenes de dumping. Dieciocho de las treinta y ocho investigaciones antidumping y sobre derechos compensatorios iniciadas por la Comisión de Comercio Internacional en los primeros ocho meses de gobierno, han sido dirigidas a las exportaciones chinas, en tanto que catorce de las cuarenta y dos órdenes emitidas por la Comisión durante el mismo periodo van dirigidas contra los exportadores chinos; por lo que, se decidió cambiar las cosas con respecto a los aranceles, presentándolas como respuesta a infracciones de propiedad intelectual en China, las cuales serían investigadas por medio de una solicitud contenida en la Ley de Comercio de 1974, este sería el tercer frente abierto sobre las relaciones comerciales entre la nación norteamericana y China (DIIS, 2017, pp. 37-39). 
Finalmente, la última promesa en la agenda comercial de Trump radica en el uso del poder presidencial para remediar las disputas comerciales, el cual consiste en la capacidad del presidente de los EE. UU. de imponer medidas o sanciones a países u organizaciones extranjeras, y que se considere hayan realizado prácticas comerciales injustificables, este privilegio se ha conoce como la sección 301 de la Ley de Comercio de 1974.

Por ello, en agosto de 2017, se informó que se estaba considerando iniciar una investigación de la Sección 301, por iniciativa propia, sobre las consecuencias comerciales de las leyes de propiedad intelectual infringidas por China. El 14 de agosto de 2017, el mandatario instruyó a la USTR a tomar en cuenta el uso del artículo 301 en concordancia con este tema, una solicitud acordada por Robert Lighthizer tiempo después. Esto puso en marcha un proceso, que incluía consultas con el Congreso, las cuales se esperaba que llevasen a una pesquisa formal sobre el tema.

Por todo lo anterior, puede decirse que la política comercial de Donald Trump representa una ruptura frente a la corriente principal de EE. UU. durante los últimos veinte años o más. Esta consiste no solo en su unilateralismo y las recias posturas mercantilistas, sino también en la naturaleza sistémica de sus intenciones (Porter, 2017). Esto implica desafiar en un grado u otro, la mayoría de los acuerdos comerciales en los que ha tomado parte, al forjar nuevos acuerdos con países que en otrora eran esquivos a la suscripción de dichos acuerdos, y ha intensificado el uso de correcciones en la gestión comercial del país.

\section{Críticas a la política comercial de Donald Trump}

Para el autor Mathias Koch, debido a la retórica de Trump, discernir una estrategia coherente sobre el comercio ha sido una tarea difícil. En su propuesta comercial, el mandatario estadounidense amenazó con aplicar un arancel del $45 \%$ a las importaciones procedentes de China, así como un arancel del $35 \%$ a las procedentes de México, al manifestar que los ingresos obtenidos por dicho accionar serían utilizados para pagar el muro fronterizo. Asimismo, entre los comentarios del mandatario, resalta que la OMC es un desastre, y sugirió que estaba dispuesto a retirar al país norteamericano de la organización en caso de que esta rechazara un curso más proteccionista para los EE. UU. bajo su dirección (Koch, 2017, p. 14).

La crítica a los déficits comerciales de EE. UU. ha sido una de las características recurrentes de Donald Trump, por ello, resulta necesario recordar que este hombre entró en la escena política en 1987 con ideas que ya planteaban en su 
momento un ataque a Japón por sus superávits comerciales (Koch, 2017, 15). Nada distante a realidades recientes, cuando para la campaña electoral de 2016, el ahora mandatario había manifestado su crítica abierta de las relaciones comerciales del país asiático con la nación norteamericana, al mencionar lo que él mismo reconocía como fallos, entre los cuales destacaba el no haber vencido a China en el comercio, a Japón con sus millones de automóviles importados, o bien, a México en la frontera y en el comercio (Rosales, 2018a).

La retórica del actual presidente implicaba que otras naciones usaban el comercio para explotar a los EE. UU., llegando a aducir que Europa se unió para vencer al país cuando se trataba de ganar dinero, pero en las negociaciones, resulta siempre ser necesario unificar a los aliados y dividir a los adversarios; y en esto Trump hace lo contrario: se sale del TPP; se sale del Acuerdo de París; golpea a la Unión Europea, México, Canadá y Japón. Si bien podría haber buscado una estrategia conjunta con la UE, NAFTA y Japón para abordar la sobre oferta china de acero, tema sobre el que existe preocupación a nivel mundial, resaltó que el país asiático "estafaba" a su nación, por lo que lo denominó un enemigo económico, por cuanto las acciones de este país han terminado por destruir industrias enteras por medio del pago de bajos salarios a los trabajadores, derivando la pérdida de miles de empleos (Summers, 2018).

Es decir, aunque el hecho de que el principio de ventaja comparativa de Ricardo postule que el libre comercio entre las naciones aumenta el bienestar para ambas partes, no implica que el comercio sea neutral para la distribución interna del bienestar en cada país, sino que es capaz de causar graves perturbaciones en el equilibrio nacional. No obstante, el modelo ricardiano resulta en una ortodoxia económica, por lo que economistas contemporáneos tienden a inclinarse por apoyar el libre comercio entre naciones, y en esto Trump ha dado cátedra de hacer lo contrario. El supuesto de dicho pensamiento radica en que el comercio es un juego de suma cero, en el que los beneficios para algunos países se combinan con perjuicios en otros países (Koch, 2017, p. 15).

Una de las teorías que puede complementar lo señalado, es el modelo HeckscherOhlin, el cual presupone que los factores de producción son móviles entre sectores, o de una manera más exacta, se puede decir que el capital y el trabajo pueden moverse sin costo alguno entre los diferentes segmentos de la economía, por lo que si un país aboga por la apertura hacia el comercio internacional, dicha teoría implica que los propietarios de recursos escasos son perjudicados mientras que los propietarios de recursos abundantes ganan, independiente de en qué sector de la economía se exploten los recursos. Si se traslada dicho precepto a la realidad estadounidense es posible inferir que los trabajadores poco calificados se ven perjudicados por el libre comercio, sin importar la industria específica en la que trabajan, mientras que los altamente calificados se benefician. 
Por otra parte, de acuerdo con Rosales:

Una estrategia negociadora errática, con amenazas estridentes, proclamación triunfalista de concesiones obtenidas e imposición de plazos breves y perentorios so pena de nuevas amenazas, es exactamente lo que jamás recomendaría cualquier serio estudioso de las experiencias de negociación con China. La estrategia de Trump es audaz y contradice radicalmente lo que propone Kissinger, por ejemplo. En la medida que esta audacia empiece a afectar los resultados de Wall Street, de las exportaciones agrícolas y las ventas automotrices, el espacio de dicha audacia se irá reduciendo. (2018b, p. 13)

Bajo las perspectivas planteadas, Mathias Koch (2017) considera que la agenda proteccionista de Trump puede obtener legitimidad económica al considerarse no solo en términos de sus efectos en la estadounidense, sino al abordar la perspectiva de los trabajadores poco calificados y las industrias que compiten con las importaciones. Por lo tanto, mientras la teoría económica contemporánea afirma que la economía estadounidense obtendría beneficios del libre comercio, no significa que este sea neutral con respecto a las maneras de distribuir el bienestar dentro del sistema. Por consiguiente, considerándose el modelo Heckscher-Ohlin, es posible pensar que el comercio tiene fuertes efectos al distribuir, al tiempo que predice que un sistema de libre comercio daña a los trabajadores poco calificados.

Por su parte, de acuerdo con Méndez-Coto:

Reconociendo la existencia de una estructura económica internacional caracterizada - siguiendo el método de conceptualización de Waltz- por la disposición de las unidades (que se deriva de sus distintas capacidades relativas), cabe pensar que los cambios en esas capacidades relativas deberían traducirse en transformaciones, cuestionamientos o consolidación de las instituciones y los regímenes internacionales prevalecientes en el ámbito de la economía política internacional. (2016, p. 16)

Por lo que, de acuerdo con ello, se observa que las acciones del presidente Trump no buscan fortalecer las instituciones y regímenes internacionales, propias de la economía, sino que como se ha expuesto hasta este punto, su interés y búsqueda de resultados se basa en "Make American Great Again", como lo esbozaba su eslogan de campaña. Además, sin duda las relaciones internacionales han tenido un remesón con el desarrollo de la política exterior (comercial) de los EE. UU. 
De acuerdo con Susan Strange, citada por Marco Méndez (2016), no se puede perder de vista el cuestionamiento de la Escuela Inglesa de la Economía Política Internacional, que por una parte, señala el carácter legitimador de la irresponsabilidad económica y financiera de los EE. UU. (p. 17); y por otra parte que, gracias a la estructura y composición de la normativa estadounidense el presidente Trump puede tomar una serie de medidas que contrastan, como se ha señalado, con la dinámica de su predecesor.

Según Porter (2017), la postura comercial de Trump se relaciona con la existencia de varias leyes en los EE. UU. que otorgan al presidente una autoridad muy amplia para regular el comercio internacional sin necesitar alguna legislación nueva o especial del Congreso. Primero, el artículo 125 de la Ley Arancelaria de 1974 brinda al mandatario autoridad para terminar un acuerdo de libre comercio, al tiempo que le otorga la potestad para aumentar los aranceles por medio de proclamaciones presidenciales que reducen los niveles arancelarios (de conformidad con el Tratado de Libre Comercio) con el fin de ejercer los derechos de EE. UU.

Otro ejemplo, recae en la Ley de Emergencia Internacional de Poderes Económicos de 1977 (IEEPA, por sus siglas en inglés), la cual autoriza al presidente a regular todas las formas de comercio internacional (y congelar activos) cada vez que el mandatario encuentre que hay alguna inusual o extraordinaria amenaza a la economía. Igualmente, la Sección 301 de la Ley de Comercio de 1974, provee al presidente de amplia autoridad para responder al comercio desleal, así como a prácticas que pueden incluir una violación del acuerdo comercial o cualquier acto, política, o práctica de un país extranjero que resulte irrazonable, o bien, llegue a gravar el comercio estadounidense (Porter, 2017).

En la actualidad, este país constituye el mercado único más grande del mundo, en el que los ciudadanos estadounidenses obtienen, según el Banco Mundial, uno de los mayores ingresos per cápita, el cual ronda los USD 59.531. Asimismo, debido a su tamaño económico, las medidas de política económica, en particular las comerciales, tienen un gran impacto. Las consecuencias de una política comercial proteccionista en los EE. UU. pueden, no solamente limitarse a las dimensiones económicas, sino que también tener implicaciones políticas y sociales importantes (Felbermayr, Steininger y Yalcin, 2017, p. 28).

Asimismo, en términos de sus aranceles, EE. UU. se puede considerar una economía muy abierta debido a que estos son bajos; este los ha reducido tanto dentro del NAFTA como dentro de la OMC, en comparación con sus respectivos socios comerciales. No obstante, si se toman en cuenta las barreras no arancelarias, esta afirmación debe ser calificada. Por ello, al examinar la protección 
comercial no arancelaria, EE. UU. demuestra ser cada vez más proteccionista, en particular en los últimos años (Felbermayr, Steininger y Yalcin, 2017, p. 29).

Según Pelkmans (2018), desde que Trump llegó al poder, la Unión Europea ha sabido que el liderazgo global en comercio e inversión debe ser perseguido por los principales comerciantes como la propia Unión Europea y China, a la vez que es probable que se les unan los once signatarios restantes del Acuerdo de Asociación Transpacífico. Lo que ha cambiado ahora es que, ya no es posible ignorar la naturaleza proteccionista e impulsiva de la nueva política comercial de los Estados Unidos. Por lo tanto, el liderazgo de la Unión Europea tendrá que combinarse con una firme valentía de oponerse y contener las inclinaciones proteccionistas del mandatario estadounidense (Pelkmans, 2018, p. 3).

\section{Primeros años de la Administración}

Una de las principales preocupaciones acerca de la política comercial, era el temor de su interés por imponer barreras comerciales, pero, aunque estas acciones podrían afectar de manera notable la economía estadounidense, parece que el verdadero objetivo del presidente consistía en buscar la forma de aumentar los empleos al tiempo que mantenía los niveles elevados de los mercados de valores actuales, por lo que tiene sentido usar las recurrentes amenazas proteccionistas para presionar a las compañías extranjeras a que inviertan en el país.

Ahora bien, de acuerdo con O'Grady (2018) hay dos riesgos potenciales para la estrategia proteccionista de Donald Trump. En primer lugar, los actos proteccionistas pueden influenciar a las naciones pequeñas para cooperar con los EE. UU., y alentar a sus empresas a invertir en el país, incluso si se reduce el empleo en el país extranjero. Sin embargo, este efecto se amplificará si se depende de EE. UU. para la seguridad, aunque sin ser tan efectivo en alguno como por ejemplo China, que es suficiente, y que por su tamaño puede rechazar el proteccionismo estadounidense. Esto podría afectar las cadenas de suministro, o bien, amenazar a los mercados financieros estadounidenses al reducir las compras de bonos del Tesoro.

Para Steinberg (2018), esto recae en el perfil mercantilista de Donald Trump. Tiene impulsos e instintos proteccionistas, ve el comercio internacional como un juego de suma cero y considera que los enemigos del déficit comercial son derrotados. En resumen, él es la antítesis de lo que durante las últimas décadas ha marcado a los líderes estadounidenses, es decir, defensores de un orden económico global basado en reglas, e inclinados a cooperar con otros gobiernos para promover los intereses mutuos a largo plazo, incluso se ven al mismo 
tiempo las ventajas para las empresas estadounidenses. No obstante, durante su primer año, sus ambiciones se han topado con la realidad de que el poder del presidente de los EE. UU., es más limitado de lo que había creído. Esto, por cuanto el sistema de controles y equilibrios del país ha llevado al mandatario a adoptar una postura más cautelosa, ejemplo de esto sería la forma en que el presidente Trump desea resolver el tema con México, mediante la imposición de un arancel o cuota de importación (Nájar, 2017).

Por otra parte, las cifras de las actividades comerciales durante el primer año de gobierno de Donald Trump distan mucho de las predicciones catastróficas que se escuchaban desde la campaña electoral. Sin embargo, un considerable aumento en las importaciones ha acarreado una serie de manifestaciones debido al déficit que esto representa, se considera como un bálsamo la notable alza que experimentaron las exportaciones durante el 2017, que alcanzaron los 1.5 billones de dólares, mientras que sus importaciones registraron los 2.4 billones de dólares (Trade Map, 2018).

Pero los efectos reales de la gestión de Trump se ponen de manifiesto al confrontar las cifras que conllevan al cálculo de la balanza comercial; que se traducen en un saldo positivo o negativo. Los siguientes datos ilustran el contraste mencionado:

Tabla 1

Saldo comercial entre los años 2015 y 2017 (valor en USD)

\begin{tabular}{|l|c|c|c|}
\hline \multicolumn{1}{|c|}{ Gobierno } & \multicolumn{2}{|c|}{ Administración Obama } & $\begin{array}{c}\text { Administración } \\
\text { Trump }\end{array}$ \\
\hline Años & 2015 & 2016 & 2017 \\
\hline Importaciones & 2313424569 & 2275391150 & 2409480182 \\
\hline Exportaciones & 1501845864 & 1451010702 & 1546732881 \\
\hline Saldo comercial & -811.578 .705 & -824380448 & -862747301 \\
\hline
\end{tabular}

Elaboración propia con base en Trade Map - International Trade Statistics (2018).

Los datos anteriormente resaltados, permiten comprobar una situación negativa con respecto al saldo comercial de los EE. UU. durante tres años consecutivos lo cual deja clara la existencia de un déficit comercial palpable y cuantificable que tendrá que ser revertido por parte del gobierno actual. No obstante, tam- 
bién podría acrecentar la tendencia al déficit, si mantiene su postura proteccionista y la recurrente retórica.

Por último, el inicio de la administración Trump, con respecto al comercio internacional, ha sido menos dañino para el Sistema de Comercio Multilateral de lo que se temía, aunque mucho de esto se debe a las barreras que el presidente norteamericano no puede pasar por alto, y al hecho de que sus constantes declaraciones de guerras comerciales y amenazas de abandono de los acuerdos comerciales no han fructificado, al menos durante su primer año al frente de la Casa Blanca. Además, esto ha permitido observar que se empleará una estrategia cada vez más agresiva y confrontativa para obtener ventajas comerciales, sin violar las reglas de la OMC, pero al mismo tiempo influir en el sistema de gobernanza basado en normas concretas, especialmente por el tipo de liderazgo de Donald Trump.

\section{Conclusiones}

La política comercial de la administración Trump se enmarca en una visión mercantilista, y no comulga con las dinámicas actuales del comercio, marcadas por posturas de libre mercado y suscripción de acuerdos comerciales administrados por las partes involucradas. Sin embargo, las tendencias mencionadas, fungen como amenazas constantes para las industrias estadounidenses, así como para la clase trabajadora, desde la perspectiva del mandatario norteamericano, por lo que su renuencia a propuestas que sugieran una apertura descuidada, no parecen ser parte de la agenda comercial. Por el contrario, es muy posible que los discursos de guerra comercial y de medidas agresivas ante otras potencias sean una constante.

Por otra parte, resulta muy claro que la agenda de los EE. UU. no contempla a América Latina en términos de prioridad. Algunas de las acciones del gobierno de Trump se avocaron al análisis de ciertos tratados comerciales suscritos con algunos de los socios hemisféricos, pero se termina por abordar tópicos relacionados con lo laboral y el medio ambiente. De igual forma, el otro acuerdo, de carácter multilateral, el CAFTA-DR, tampoco ocupa un estatus de prioridad dentro de la hoja de ruta del actual gobierno, por lo que es posible pensar que EE. UU. se limitará a la tutela y administración del acuerdo mientras no se lesionen los intereses propios, ni se comprometan las condiciones de los países firmantes. Una realidad muy diferente a las relaciones comerciales que mantiene los EE. UU. con otras potencias y países de otras latitudes, de las que se espera sus interacciones sean mucho más hostiles. 
En este tema es posible pensar en China -país al cual le declaró la guerra comercial-, Japón, y la misma Unión Europea, cuya postura de Trump los coloca como entes capaces de socavar la economía de los EE. UU., así como de provocar efectos negativos sobre los exportadores, la clase trabajadora, la industria, las divisas, además de imponer barreras comerciales que limitan el ámbito de actividad comercial para el país desde la visión del presidente norteamericano. Por todo esto, se puede decir que las relaciones terminarán por acarrear más tensiones en el plano comercial, en tanto que los procesos en las instancias supranacionales también terminarán por acaparar la agenda de las instituciones en aras de buscar la mejor salida a las potenciales controversias, así como consecuencias y sanciones que esta situación presupone.

El seguimiento de las reglas y las normas internacionales relacionadas con el comercio es otra de las encrucijadas que presupone la gestión de Trump. Resulta necesario recordar que la postura del presidente norteamericano no es la más afable con la OMC, por cuanto ha sido capaz de arremeter en su contra, al amenazar incluso, con abandonarla bajo el supuesto de contribuir a los intereses de otras naciones para imponer condiciones desleales a los EE. UU. Por tanto, es claro que la gobernanza global en términos de procesos supranacionales no responde a los intereses inmediatos de Donald Trump, por lo que su postura no cambiará en el corto plazo.

La gestión de Trump termina con un déficit en su saldo comercial durante su primer año de gobierno. En este sentido, lo que cabe decir, por ahora, es que la postura proteccionista asumida por el mandatario y su agenda comercial debe empezar a revertir las estadísticas actuales, o bien, terminará consolidando, no solo una posición impopular, sino que, en el caso de no lograr cambiar las condiciones de déficit, podría terminar por alentar su incremento, hasta generar el escenario desastroso que la mayoría de sus detractores han tratado de anticipar. Ante esto, es muy importante considerar que el primer año de gobierno de Trump no marca por sí mismo la ruta que puede seguir, pero en un escenario en el cual la guerra económica con China tome un rumbo desfavorable, y en el que Estados Unidos continúe aislándose de sus socios comerciales, será posible pensar en escenarios que implican una peligrosa propensión a la crisis.

Finalmente, pese a que la gestión comercial llevada a cabo por parte del presidente Trump no ha comprometido la dinámica del comercio internacional, resulta evidente su intención de influir de manera directa en la forma en que estos intercambios se realizan. No obstante, suficiente tiempo habrá para determinar, ya sea por asimilar las decisiones tomadas por el mandatario norteamericano, o bien, por comprobar si la estrategia del actual gobierno logrará salvaguardar de la mejor forma los intereses nacionales de los EE. UU. La carrera de Trump por cambiar el comercio internacional apenas comienza. 


\section{Referencias}

BBC. (2017). ¿Por qué la decisión de Trump de retirar a EE.UU. del Acuerdo Transpacífico (TPP) es una gran noticia para China? Recuperado de: https:// www.bbc.com/mundo/noticias-internacional-38063522

Bellina, J. y Frontons, G. (2012). Política comercial, acuerdos y negociaciones externas: La Argentina y el MERCOSUR. INVENIO. Recuperado de: https://www. google.com/url?sa=t\&source=web\&rct=j\&url=https://dialnet.unirioja.es/descarga/articulo/4208250.pdf\&ved=2ahUKEwiw8vmvzr_1AhUDuHEKHV-LD 6E4ChAWMAJ6BAgGEAI\&usg=AOvVaw210IPhNJLbZJAt90sm4QTO

Castorena, C.; Gandásegui, M. y Morgenfeld, L. (2018). Estados Unidos contra el mundo Trump y la nueva geopolítica. Buenos Aires: CLACSO.

Chorev, N. (2012). International trade policy under George W. Bush. En A. Wroe $y$ J. Herbert. Assessing the George W. Bush Presidency: A tale of two terms, (pp. 129-146). Edinburgo: University Press.

CINPE. (2009). La política comercial en los primeros 100 días de Obama. Puentes entre el comercio y el desarrollo sostenible, 10(2). Recuperado de: http://biblioteca.icap.ac.cr/BLIVI/COLECCION_UNPAN/BOL_JULIO_2013_64/ CINPE/2009/puentes10_2_1.pdf

CNN. (2019). Gobierno de Trump califica a China de manipulador de divisas. Recuperado de: https:/cnnespanol.cnn.com/2019/08/05/gobierno-de-trumpcalifica-a-china-de-manipulador-de-divisas

Crook, C. (2003). George W. Bush's biggest failure is trade policy. The Atlantic. Recuperado de: https://www.theatlantic.com/politics/archive/2003/12/ george-bushs-biggest-failure-is-trade-policy/377248/

Danish Institute for International Studies (DIIS). (2017). U. S. trade policy under Trump: Assessing the unilateralist turn. Copenhague: DIIS.

DIRECON. (2015). Análisis de las relaciones comerciales entre Chile y Estados Unidos en el marco del tratado de libre comercio. Recuperado de: http:// www.sice.oas.org/TPD/CHL_USA/Studies/00-INFORME-EEUU-Mayo-2015.pdf

EFE. (2017). Abe y Trump preparan su primera cumbre en Washington para febrero. Recuperado de: https://www.efe.com/efe/espana/portada/abe-y-trumppreparan-su-primera-cumbre-en-washington-para-febrero/10010-3160048

Felbermayr, G.; Steininger, M. y Yalcin, E. (2017). Quantifying Trump: The costs of a protectionist US. Cesifo Forum, 18(4), 28-36. 
France24. (2018). Japón y Estados Unidos formalizan negociaiones para acuerdos comerciales. Recuperado de: https://www.france24.com/es/20180927economia-japon-estados-unidos-acuerdo

Froman, M. (2017). Trade, growth and jobs: U.S. trade policy in the Obama Administration. Recuperado de: http://americastradepolicy.com/trade-growthand-jobs-u-s-trade-policy-in-the-obama-administration/\#.XYEB5C3SHOQ

Gerwin, E. (2015). The Obama trade agenda: Five things for progressives to like. Recuperado de: https://www.progressivepolicy.org/wp-content/ uploads/2015/02/2015.02-Gerwin_The-Obama-Free-Trade-Agenda.pdf

Gordon, N. (2017). Trump's trade policy: Separating the normal from the dangerous. Recuperado de: https://www.cer.eu/sites/default/files/insight NG_10.11.17.pdf

Koch, M. (2017). Trump-trade in historical perspective: Three lessons from the history of $U$. S. trade policy (Tesis de maestría). Universiteit Utrecht, Países Bajos.

La Nación. (2017). La antilógica comercial de Trump. Recuperado de: https://www.nacion.com/opinion/foros/la-antilogica-comercial-detrump/6LCO3LCU6JAGLLZIBJ236XZUAA/story/

Méndez, M. (2016). Multilateralismo, gobernanza y hegemonía en la estructura económica internacional: del G7 al G20. Relaciones Internacionales, 31: 13-32.

Nájar, A. (16 de agosto de 2017). 5 propuestas de Trump para renegociar el NAFTA... que México difícilmente aceptará. BBC. Recuperado de: https://www. bbc.com/mundo/noticias-america-latina-40833540

New York Times. (2018). Los negociadores que intentan rescatar el TLCAN. Recuperado de: https://www.nytimes.com/es/2018/09/22/tlcan-freelandlighthizer-trump

O'Grady, B. (2018). Trump and trade: The first year. Advisor perspectives. Recuperado de: https://www.advisorperspectives.com/commentaries/2018/02/06/ trump-trade-the-first-year

Oficina Económica y Comercial de España en Washington. (2015). Informe Económico y Comercial de los Estados Unidos. Recuperado de: http://www. comercio.gob.es/tmpDocsCanalPais/9F547F8FFC045F68EE18DD8C51E DE861.pdf

OMC. (2018). Examen de las políticas comerciales. Informe de los Estados Unidos. $\mathrm{WT} / \mathrm{TPR} / \mathrm{G} / 382.12$ de noviembre.

Pelkmans, J. (2018). Trade Policy-Making Under Irrationality. Bruselas: CEPS.

Pollin, R. (2005). Los contornos del declive. Madrid: Ediciones Akal S. A. 
Porter, D. (2017). A new era for U.S. trade policy: what to expect from Trump Trade. Recuperado de: https://www.iccmex.mx/uploads/Curtis\%20PPT\%20 for $\% 20$ Mexico $\% 20$ City $\% 20$ Meetings.pdf

Rosales, O. (2018a). Las claves del conflicto económico China-Estados Unidos. Flacso Chile. Recuperado de: http:/www.flacsochile.org/slider/las-clavesdel-conflicto-economico-china-estados-unidos

Rosales, O. (2018b). Guerra Comercial. Economía y Administración, Especial (173), 6-14. Recuperado de: http://www.fen.uchile.cl/uploads/images/files/ revista_173(2).pdf

Sistema Económico Latinoamericano y del Caribe. (2009). Aspectos de la política económica y comercial bajo la Administración Obama y sus implicaciones para América Latina y el Caribe. Seminario regional sobre las relaciones económicas entre Estados Unidos y América Latina y el Caribe en un contexto de crisis global. Caracas, Venezuela.

Steinberg, F. (2018). A year of Trump and trade policy. Recuperado de: http:// www.realinstitutoelcano.org/wps/wcm/connect/f135bae0-eb4c-47d2b130-9f893b8ea917/Commentary-Steinberg-Year-Trump-trade-policy.pdf?MOD=AJPERES\&CACHEID=f135bae0-eb4c-47d2-b1309f893b8ea917

Summers, L. (2018). Donald Trump`s trade policy violates every rule of strategy, Financial times. Recuperado de: https://www.ft.com/content/4eda86ec67da-11e8-aee1-39f3459514fd

USTR. (2008). The President's trade agenda. Recuperado de: https://ustr.gov/aboutus/policy-offices/press-office/reports-and-publications/archives/2008/2008trade-policy-agenda-and-2007-annua

USTR. (2017). U. S. President's 2017 trade policy agenda and 2016 annual report of the President of United States on the Trade Agreements Program. Recuperado de: https://ustr.gov/about-us/policy-offices/press-office/reports-andpublications/2017/2017-trade-policy-agenda-and-2016

USTR. (2018). U. S. President's 2018 trade policy agenda and 2017 annual report of the President of United States on the Trade Agreements Program. Recuperado de: https:/ustr.gov/about-us/policy-offices/press-office/reports-andpublications/2018/2018-trade-policy-agenda-and-2017

VOA. (2017). Trump elogia a Malasia por inversiones en EE. UU. Recuperado de: https://www.voanoticias.com/a/trump-elogia-primer-ministro-malasia/4025911.html

Wolff, M. (2018). Fire and fury. Inside the Trump White House. New York: Henry Holt and Company. 